\title{
Elements and ephemeris of planet 1908 DC.
}

The following elements were computed from observations on April 26, May 23, June r, June 5, 1908. The first is a photographic observation furnished by Rev. Focl $H$. Metcalf, the others are by Mr. M. Frederickson.

Elements.

1908 April 26.5 Greenw. m. t.

$$
\begin{aligned}
& M=22^{\circ} 46^{\prime} 3^{\prime \prime} \\
& \omega=3453^{6} 5
\end{aligned}
$$

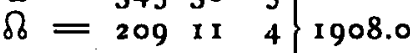

$$
\begin{aligned}
& i=195^{6} 6 \\
& \varphi=65225 \\
& \mu=612: 32 \\
& \log a=0.50869
\end{aligned}
$$

\begin{tabular}{|c|c|c|c|}
\hline $\begin{array}{r}1908 \\
\text { April }\end{array}$ & & $\begin{array}{c}a \\
-0.0\end{array}$ & $\begin{array}{l}3 \\
+5^{\prime \prime}\end{array}$ \\
\hline May & 19 & +0.2 & +6 \\
\hline & 23 & +0.4 & +3 \\
\hline & 25 & -0.2 & +4 \\
\hline June & I & +0.3 & +2 \\
\hline & 5 & +0.5 & $+I$ \\
\hline
\end{tabular}

Residuals $(\mathrm{O}-\mathrm{C})$.

Heliocentric coordinates rgo8.0. $x=[9.99392] \cdot r \cdot \sin \left(v+283^{\circ} 18^{\prime} 11^{\prime \prime}\right)$

\begin{tabular}{|c|c|c|c|c|c|}
\hline 1909 & \multicolumn{2}{|c|}{$\alpha(1909.0)$} & $\delta(1909.0)$ & $\log A$ & Ab.-T. \\
\hline une $I$ & $20^{h} 47^{m}$ & ${ }^{2} 3^{*}$ & $+8^{\circ} 24^{\prime} 3$ & $0.419 \times 3$ & $2 \mathrm{I}^{\mathrm{m}} 50^{\circ}$ \\
\hline $\mathbf{I}$ & 46 & 17 & 843.3 & $4134^{\circ}$ & 33 \\
\hline 2 & 44 & 53 & 90.0 & 40793 & 17 \\
\hline 2 & 43 & I I & 914.0 & 88 & 2 I 2 \\
\hline aly & 41 & 14 & 925.2 & 39829 & 2049 \\
\hline & $\begin{array}{l}39 \\
36\end{array}$ & & $\begin{array}{ll}9 & 33.4 \\
9 & 38.4\end{array}$ & $\begin{array}{l}39419 \\
39064\end{array}$ & $\begin{array}{l}37 \\
27\end{array}$ \\
\hline I & 34 & 9 & 940.1 & 38765 & 18 \\
\hline $\mathbf{I}$ & 3 I & 28 & 938.5 & 38529 & 12 \\
\hline 2 & 28 & 41 & 933.6 & $3^{8362}$ & 7 \\
\hline 2 & 25 & 50 & 925.3 & 38265 & 4 \\
\hline 2 & 22 & $5^{8}$ & 914.0 & 38232 & 3 \\
\hline ug. & & 8 & 859.6 & 38275 & 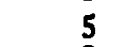 \\
\hline & 17 & 21 & 842.3 & 38392 & 8 \\
\hline $\mathbf{I}$ & 14 & 42 & 822.4 & $3^{8} 5^{86}$ & $\mathbf{I}_{3}$ \\
\hline $\mathbf{I}$ & $\mathbf{I} 2$ & IO & 0.1 & 38844 & 20 \\
\hline $\mathbf{I}$ & & 49 & 735.6 & $39 \times 68$ & 30 \\
\hline 2 & 7 & $4 I$ & $7 \quad 9.4$ & 39556 & $4 I$ \\
\hline 2 & & 48 & 641.7 & 40006 & $20 \quad 54$ \\
\hline 3 & 4 & I I & 613.0 & 40516 & 218 \\
\hline ept. & 20 & 54 & +543.7 & 0.41086 & $2 I 25$ \\
\hline
\end{tabular}
$y=[9.99777] \cdot r \cdot \sin (v+1921921)$ $z=[9.28904] \cdot r \cdot \sin (\nu+2513123)$

University of Minnesota, Minneapolis, Minn., I 909 May $\$ 5$.
Ephemeris $12^{\text {h }}$ Gr.m.t.

Opposition in $\alpha$ July 27.

Magnitude at opposition 13.1.

K. Burns, L. W. McKeclean.

Beobachtungen des Planeten 1908 EP

am Utrechter Refraktor (Öffnung $=26 \mathrm{~cm}$, Fokallänge $=3 \mathrm{Ig} \mathrm{cm}$, Vergr. $=94$ ).

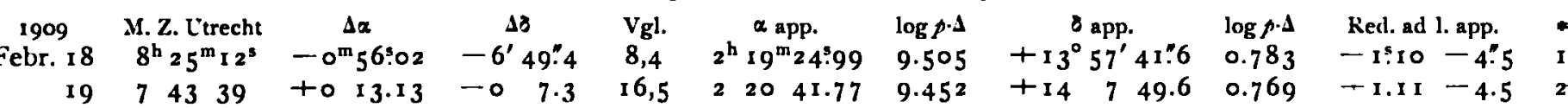

Mittlere Örter der Vergleichsterne.

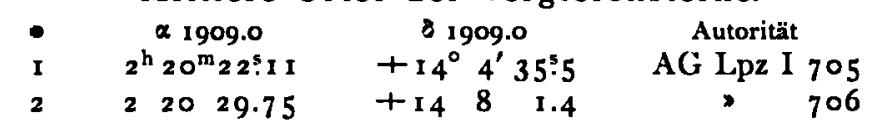

Utrecht, I 909 März I o.

\section{Notiz betr. Var. 17.1909 Draconis.}

Der Veränderliche BD $+5^{\circ}{ }_{17} 8_{2}$ findet sich in der normalen Größe $9^{m}$ noch auf zwei Platten, die 1909 April 10 $9^{\mathrm{h}} 53^{\mathrm{m}}-1 \mathrm{1}^{\mathrm{h}} 35^{\mathrm{m}}$ M. Z. Königstuhl exponiert sind; ebenso auf zwei Platten von 1909 Mai $2010^{\mathrm{h}} 14^{\mathrm{m}}-12^{\mathrm{h}} 14^{\mathrm{m}}$ M. Z. Königstuhl. Dagegen erreicht er auf einer Aufnahme 1909 Mai $189^{\mathrm{h}} 30^{\mathrm{m}} \cdot 5-\mathrm{I}^{\mathrm{h}} 5^{\circ} \cdot 5 \mathrm{Kgst}$. diese Helligkeit nicht; er ist bedeutend heller als am 8. Mai, aber doch nur etwas heller als $10^{\mathrm{m}}$.

Astrophys. Institut Königstuhl-Heidelberg, I 909 Mai 2 I.

M. Wolf.

Inhalt zu Nr. 4326. H. Seeliger. Über das Eindringen eines Weltkörpers in eine kosmische Staubwolke. 8r. - F. Scheiner. Über die Resultate der Beratungen der diesjährigen astrophotographischen Konferenz zu Paris. 89. - $I$. Luther. (444) Gyptis. 89. M. Frederickson. Elements and ephemeris of minor planet $1908 \mathrm{CC}$. 91. - M. W. Whituey and C. E. Furness. Observations of long-period variables. 91. - W. Krebs. Zwei merkwdirdige Leonidenschweife. 93. $-K$. Burns, L. W. I/VASeelean. Elements and ephemeris of planet 1908 DC. 95. - F. van der Bill. Beobachtungen des Planeten 1908 EP. 95 . - M. Wolf. Notiz betr. Var. 17.1909 Draconis. 95.

(ieschlossen 1909 Juni 4. Herausgeber: H. Kobold. Druck von C. Schaidt (G. \& A. Oheim). Expedition: Kiel, Moltkestr. 8o. 